\title{
SOME RESULT OF NON-COPRIME GRAPH OF INTEGERS MODULO n GROUP FOR n A PRIME POWER
}

\author{
Masriani $^{1}$, Rina Juliana ${ }^{2}$, Abdul Gazir Syarifudin ${ }^{3}$, I Gede Adhitya Wisnu \\ Wardhana $^{4^{*}}$, Irwansyah ${ }^{5}$ and Ni Wayan Switrayni ${ }^{6}$ \\ 1,2,3,4,5,6 Universitas Mataram, Mataram, Indonesia \\ Email : ${ }^{1}$ masriani@unram.ac.id, ${ }^{2}$ rinajuliana@unram.ac.id, ${ }^{3}$ abdulgazirs@gmail.com, \\ 4adhitya.wardhana@unram.ac.id, 5irw@unram.ac.id, ${ }^{6}$ niwayan.switrayni@unram.ac.id \\ *Penulis Korespondensi
}

\begin{abstract}
One interesting topic in algebra and graph theory is a graph representation of a group, especially the representation of a group using a non-coprime graph. In this paper, we describe the non-coprime graph of integers modulo $n$ group and its subgroups, for $n$ is a prime power or $n$ is a product of two distinct primes.
\end{abstract}

Keywords: group, integer module, non-coprime.

\section{INTRODUCTION}

The non-coprime graph of a finite group was introduced by Mansoori et al. [1]. In [1], the authors determined some numerical invariants of the non-coprime graph of a finite group, such as its diameter, girth, dominating number, independence, and chromatic number. Moreover, they characterize the planar non-coprime graph of a group and the regular noncoprime graph of a nilpotent group. Furthermore, they also stated a connection between the non-coprime graphs and some prime graphs.

Aghababaei-Beni and Jafarzadeh [2] investigated the properties of Cartesian and tensor products of non-coprime graphs of finite groups such as the dihedral and semi-dihedral groups. They considered the properties such as the independence, clique, chromatic number, covering number, diameter, connectedness, and the existence of the Eulerian spanning subgraph. They also gave a characterization for such graphs to be an Eulerian graph and to be a planar graph. Recently, Aghababaei et al. [3] extended some results in [2]. They studied the non-coprime of a finite group with respect to a subgroup and investigated some properties of such a graph, including its diameter, chromatic number, clique, and the number of connected components. They also investigated some properties of the non-coprime graph of the nilpotent group.

Some authors give some properties of the non-coprime graph and the coprime graph to more specific groups. Rilwan et al. give some properties of the non-coprime graph of integer [4], Juliana et al. give some properties of the non-coprime graph of an integer modulo [7], and Syarifudin et al. give some properties of the non-coprime graph of dihedral groups [8].

In this paper, we describe the non-coprime graph of the group $\mathbb{Z}_{n}$ and that of its subgroups, where $n$ is a prime power or $n$ is a product of two distinct primes. We used the result of the coprime graph of the group $\mathbb{Z}_{n}$ as the non-coprime graph is the duality of the coprime graph [6]. This paper is organized as follows. Section 2 (Some Basic Notions) collects some basic 
notions related to group and graph. We give our main results in Section 3 (Main Results). Some concluding remarks are collected in Section 4 (Conclusions). Finally, we give some related references in the References section.

\section{SOME BASIC NOTION}

Let $G$ be a finite group and $|G|$ be the number of elements in $G$ or the order of $G$. The definition of the order of an element in $G$ is as follows.

Definition 1. Let $G$ be a finite group with the identity element $e$. The order of $g \in G$, denoted by $|g|$, is the smallest positive integer $n$ such that $g^{n}=e$.

Let $H$ be any subgroup of $G$. In the rest of the paper, if $H$ is a subgroup of $G$, then we denote it by $H \leq G$. Also, let $a$ be an element in $G$. A subgroup $<a>=\left\{a^{n} \mid n \in \mathbb{Z}\right\}$ is called a cyclic subgroup of $G$ generated by an element $a$. The following theorem states a relation between $|H|$ and $|G|$.

Theorem 1. (Lagrange's Theorem [4]). If $G$ is a finite group and $H \leq G$, then $|H|$ is a divisor of $|G|$.

As a consequence of Theorem 1 , we have that $|\langle g\rangle|$ divides $|G|$.

A graph is one crucial object in mathematics, especially in discrete mathematics and its applications. The definition of a graph is as follows.

Definition 2. [5]. A graph is a pair $\Gamma=(V, E)$, where $V$ is a non-empty set of vertices, and $E \subseteq V \times V$ is a set of edges.

We have to note that, in the rest of the paper, we only use a simple undirected graph, i.e., we assume that $\left(v_{i}, v_{j}\right)=\left(v_{j}, v_{i}\right)$ for all $\left(v_{i}, v_{j}\right) \in E$.

Definition 3. An undirected graph $\Gamma$ is complete if for any $v_{i}, v_{j} \in V$, we have that $\left(v_{i}, v_{j}\right) \in$ E. If $|V|=m$, then we denote an undirected complete graph $\Gamma$ as $K_{m}$.

Let $a$ and $b$ be two integers. The greatest common divisor of $a$ and $b$ usually denoted by $(a, b)$. The following definition defines the non-coprime graph of a finite group.

Definition 4. [1]. Let $G$ be a finite group. The non-coprime graph of $G$ denoted by $\bar{\Gamma}_{G}$, is a graph whose vertices are all elements of $G \backslash\{0\}$. Two different vertices $x$ and $y$ in $\bar{\Gamma}_{G}$ are adjacent if $(|x|,|y|) \neq 1$.

\section{MAIN RESULT}

Let $\mathbb{Z}_{n}=\{0,1, \ldots, n-1\}$ be the group of integers modulo $n$ with addition $(\bmod n)$ operation. The following proposition gives the non-coprime graph of $\mathbb{Z}_{n}$ when $n$ is a prime number. 
Proposition 1. If $n$ is a prime number, then the non-coprime graph of $\mathbb{Z}_{n}$ is a complete graph.

Proof. Since $n$ is a prime number, we have that $|i|=n$, for all $i=1,2, \ldots, n-1$. So, $(|a|,|b|) \neq 1$, for all, $b \in \mathbb{Z}_{n}$. These imply, $a$ and $b$ are adjacent in $\bar{\Gamma}_{\mathbb{Z}_{n}}$ for all, $b \in \mathbb{Z}_{n}-$ $\{0\}$. Therefore, the non-coprime graph of $\mathbb{Z}_{n}$ is a complete graph $K_{n-1} \cdot \square$

Here is an example of Proposition 1.

Example 1. Let $\mathbb{Z}_{7}=\{0,1,2,3,4,5,6\}$. As we can see, $|1|=7,|2|=7,|3|=7,|4|=7,|5|=$ $7,|6|=7$. So, we have that $(|a|,|b|) \neq 1, \forall a, b \in \mathbb{Z}_{7}$. Therefore, every non-zero element of $\mathbb{Z}_{7}$ are adjacent to each other. We can see $\bar{\Gamma}_{\mathbb{Z}_{7}}$ in Figure 1 .

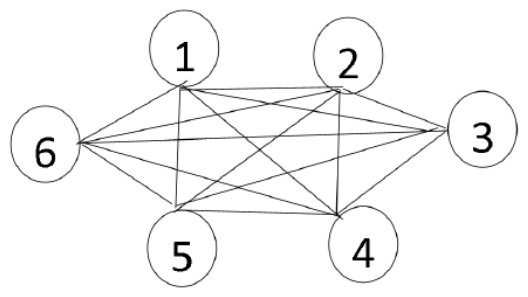

Figure 1. Non-coprime graph of $\mathbb{Z}_{\mathbf{7}}$

Let $n=p^{s}$ for some prime number $p$ and a natural number $s \geq 2$. The following theorem describes the non-coprime graph of $\mathbb{Z}_{n}$, when $n=p^{s}$.

Theorem 2. If $n=p^{s}$, for some prime number $p$ and natural number $s \geq 2$, then the noncoprime graph of $\mathbb{Z}_{n}$ is a complete graph.

Proof. Let $a$ be an element in $\mathbb{Z}_{p^{s}}$ with $\left(p^{s}, a\right) \neq 1$. The element $a$ can be written as $a=p^{k} q$ , for some $1 \leq k<s$ and an integer $q$, where $(p, q)=1$. As a consequence, we have that $|a|=p^{s-k}$. Also, for any $b \in \mathbb{Z}_{p^{s}}$ with $\left(p^{s}, b\right)=1$, we have that $|b|=p^{s}$. These imply $(|a|,|b|) \neq 1$, for all, $b \in \mathbb{Z}_{p^{s}}-\{0\}$. So, $a$ and $b$ are adjacent in $\bar{\Gamma}_{\mathbb{Z}_{p^{s}}}$ for all, $b \in \mathbb{Z}_{p^{s}}-\{0\}$. Therefore, the non-coprime graph of $\mathbb{Z}_{p^{s}}$ is a complete graph $K_{p^{s-1}} \cdot \square$

Here is an example of Theorem 2

Example 2. Let $\mathbb{Z}_{3^{2}}=\{0,1,2,3,4,5, \ldots, 8\}$. As we can see, $|1|=9,|2|=9,|3|=3,|4|=$ $9,|5|=9,|6|=3,|7|=9,|8|=9$. Consequently, we have that $a$ and $b$ are adjacent in $\bar{\Gamma}_{\mathbb{Z}_{3}{ }^{2}}$ for all $a, b \in \mathbb{Z}_{3^{2}}-\{0\}$. The non-coprime graph of $\mathbb{Z}_{3^{2}}$ is shown in Figure 2.

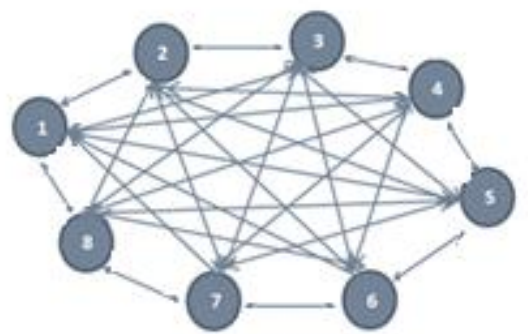

Figure 2. Non-coprime graph of $\mathbb{Z}_{9}$ 
Let $n$ be a product of two distinct primes. The following theorem describes the non-coprime graph of $\mathbb{Z}_{n}$, when $n$ is a product of two distinct primes.

Theorem 3. Let $=p_{1} p_{2}$, where $p_{1}, p_{2}$ are two distinct primes. If $H$ is a proper subgroup of $\mathbb{Z}_{n}$, then the non-coprime graph of $H$ is complete.

Proof. Let $H$ be any proper subgroup of $\mathbb{Z}_{n}$. By Theorem 1 (Lagrange's Theorem), we have that $|H|=p_{1}$ or $|H|=p_{2}$. Therefore, by Proposition 1, we have that $\Gamma_{H}$ is a complete graph.

Here is an example of Theorem 3.

Example 3. Let $\mathbb{Z}_{15}=\{0,1,2, \ldots, 14\}$. We can check that non-trivial subgroups of $\mathbb{Z}_{15}$ are $\langle 3\rangle$ and $\langle 5\rangle$. Moreover, we can see that $\langle 3\rangle=\{0,3,6,9,12\}$ and $\langle 5\rangle=\{0,5,10\}$. The noncoprime graphs of $\langle 3\rangle$ and $\langle 5\rangle$ are shown in Figure3.
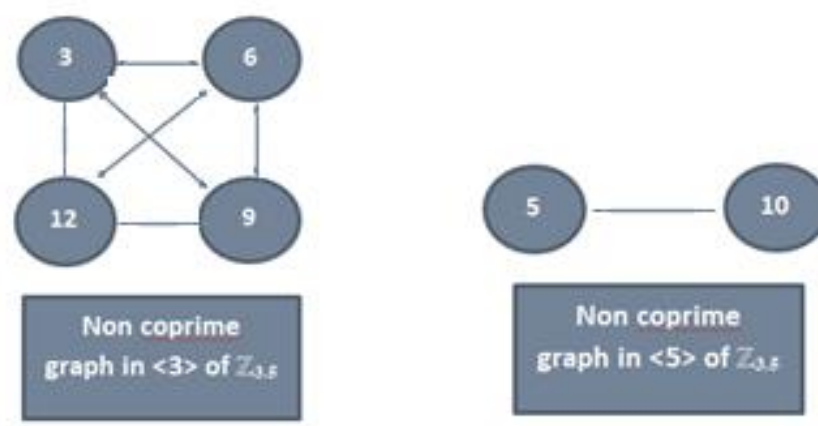

Figure 3. Non-coprime graph of subgroups in $\mathbb{Z}_{15}$

\section{CONCLUSIONS}

We have shown that the non-coprime graph of $\mathbb{Z}_{n}$, when $n$ is a prime power, is a complete graph $K_{n-1}$. Moreover, when $n$ is a product of two distinct primes, the non-coprime graphs of its non-trivial subgroups are complete graphs.

\section{REFERENCE}

[1] F. Mansoori, A. Erfanian, and B. Tolue, "Non-coprime graph of a finite group", AIP Conference Proceedings 1750, 050017, 2016.

[2] G. Aghababaei-Beni and A. Jafarzadeh, "The non-coprime graph of finite groups", Math. Interdisc. Res.3, pp. 109-119, 2018.

[3] G. Aghababaei, A.R. Ashrafi, and A. Jafarzadeh, "The non-coprime graph of a finite group with respect to a subgroup", Italian Journal of Pure and Applied Mathematics42, pp. 25-35, 2019.

[4] N. M. Rilwan, M. M. Sameema and A. R. Oli, "Non-Coprime Graph of Integers", International Journal of Mathematics Trends and Technology (IJMTT), Vol. 66 No. 2, 2020. 
[5] R.L. Roth, “A history of Lagrange's Theorem on groups”, Mathematics Magazine Vol. 74 No. 2, pp. 99-108, 2001.

[6] R. Diestel, "Graph Theory”, New York: Springer-Verlag, 2000.

[7] R. Juliana, M. Masriani, I. G. A. W. Wardhana, N. W. Switrayni, I.,Irwansyah, "Coprime Graph of Integers Modulo n Group And Its Subgroups," Journal of Fundamental Mathematics and Application Vol.3 No.1, pp 15-18, 2020

[8] Syarifudin A G, Wardhana I G A W, Switrayni N W, \& Aini Q, "Some Properties of Coprime Graph of Dihedral Group When is Prime Power", Journal of Fundamental Mathematics and Applications, vol. 3, no. 1, pp. 34-38, 2020. 